Mediterránea Ser. Biol. (1991), n. 13. Pág. 15-26

\title{
UNA NUEVA APROXIMACIÓN METODOLÓGICA AL ESTUDIO DE LAS COMUNIDADES GIPSÍCOLAS IBÉRICAS ${ }^{1}$
}

\author{
por \\ AGUSTÍN RUBIO(**), ADRIÁN ESCUDERO(*), \\ SANTIAGO PAJARÓN(*) Y ANDRÉS MOLINA(*)
}

\section{RESUMEN}

Durante los ûltimos años hemos asistido a una intensa producción de cartografía de distribución de táxones a escalas medias y grandes. Esto ha permitido plantear ciertos trabajos de síntesis biogeográfica, generalmente basados en técnicas multivariantes de clasificación. En nuestro caso hemos utilizado una técnica de ordenación (Análisis de Correspondencias Canónico), que al tiempo nos ha permitido comparar directamente la influencia de algunas variables ambientales en los grupos reconocidos. Como ejemplo hemos estudiado las relaciones existentes entre una serie de táxones gipsícolas en la Península Ibérica.

\begin{abstract}
A new Metodological Approach to the Iberian Gipsicolous Communities Study. During the last years a numerous cartographic production about taxa distribution in medium and large scales has appeared. This event has allowed the realization of some biogeographical syntesis works based usually in multivariate classification techniques. In this work we have used an ordination technique (Canonical Corespondence Analysis), that permited us to compare the influence of some environmental variables in the defined groups. As an example we have studied these relationships in some gypsicolous taxa from the Iberian Peninsula.
\end{abstract}

KEY WORDS: Canonical ordination, Corology, Gipsicolous plants, Phytosociology, Spain.

(*) Dpto. Biología Vegetal I. Fac. Biología. Universidad Complutense Madrid.

(**) Dpto. Silvopascicultura. E.T.S.I. Montes. Universidad Politécnica Madrid.

${ }^{\prime}$ Trabajo financiado en parte con cargo al proyecto de investigación E n. ${ }^{\circ} \mathrm{C} 193 / 91$ de la Comunidad Autónoma de Madrid. 


\section{INTRODUCCIÓN}

Hasta la actualidad la integración de información cartográfica en la Península Ibérica se ha realizado desde diferentes enfoques. Por un lado se han desarrollado métodos de planificación territorial (RAMOS \& AYUSO, 1974; ELENA ROSELLO \& BUNCE, 1984) con el fin de estimar los recursos reales y potenciales de una región dada. En tales casos los atributos de cada unidad geográfica considerada han sido fundamentalmente abióticos (climáticos, litológicos, etc.).

Por otro lado se han propuesto sectorizaciones biogeográficas (SAÍNZ \& HERNÁNDEZ BERMEJO, 1984; MORENO SAIZ, 1990). En estos casos la información procesada ha sido exclusivamente corológica.

Los métodos comentados suelen tener en común como fundamento metodológico, diferentes técnicas de análisis multivariante de clasificación.

El objeto de este trabajo es presentar un método, que utilizando ambos conjuntos iniciales de datos permita que la información corológica se relacione directamente con los gradientes ambientales que caracterizan un territorio. De esta forma se puede obtener información geobotánica, altamente contrastada.

\section{MÉTODO}

En primer lugar es necesario elegir el tamaño de las unidades geográficas que se utilizarán. En nuestro caso son cuadrículas U.T.M. de $10 \times 10$ $\mathrm{km}$., que es la malla más frecuentemente utilizada en trabajos corológicos (REY, 1984; FERNÁNDEZ CASAS, 1985).

Para desarrollar el método es necesario partir de dos conjuntos de datos. En primer lugar uno que sirva de base a una matriz biótica en la que a cada unidad geográfica le corresponda un valor cualitativo; normalmente la cartografía corológica no permite utilizar otra información, aunque es evidente que si existiese, los resultados obtenidos serían más satisfactorios. $\mathrm{Y}$ otro para formar otra matriz en la que cada cuadrícula se caracteriza para cada una de las variables ambientales consideradas en el estudio. En nuestra experiencia hemos podido comprobar que de la información cartografiada que se dispone las escalas más adecuadas a nuestro propósito son de $1: 1.000 .000$.

Sin duda alguna la forma más generalizada de abordar dichas ordenaciones ha sido a partir de matrices de contingencia. En este sentido hemos asistido a una gran profusión de índices de semejanza, algunos de los cuales han resultado ser de gran eficacia en estudios biogeográficos (KENKEL \& BOOTH 1987). Sin embargo, en éstos, es necesario partir de una sectorización previa que se quiere probar y sólo en un segundo paso se puede contrastar con la información abiótica (HNATIUK \& MASTIN, 1988).

Nuestra intención es abordar simultáneamente ambos conjuntos de datos. En este sentido utilizaremos técnicas de gradiente directo (WHITTAKER, 1967; JONGMAN et al., 1987; TER BRAAK \& PRENTICE, 1987). 
La ordenación de dos conjuntos de datos estructurados es posible mediante el uso de análisis canónicos (WILLIANS, 1983; GITTINS, 1985; JOGNMAN \& al., 1987). En particular, el análisis canónico de correspondencias (CCA - TER BRAAK, 1986; 1987a), el cual está disponible en paquetes informáticos enfocados a la resolución de problemas ecológicos (CANOCO -TER BRAAK, 1987b), da resultados muy satisfactorios. Parece evidente que estas técnicas están ganando rápidamente en popularidad (OKSANEN \& HUTTENEN, 1989), y ya ha sido probado su utilidad en la resolución de problemas biogeográficos (YOCCOZ \& CHESSEL, 1988).

La utilización de una forma canónica del análisis de correspondencias se debe a que la longitud de los primeros ejes extraídos en SD (unidades de desviación típica - HILL 1979) es de aproximadamente 10 SD. Si el gradiente fuese más corto cabría la posibilidad de utilizar métodos que asuman una relación linear. Para evitar el efecto de arco (HILL \& GAUCH, 1980) observado hemos optado por utilizar una técnica de corrección - detrending - mediante polinomios de $2 .^{\circ}$ grado, en lugar de la propuesta originalmente por HILL \& GAUCH (1980), debido a la inestabilidad del método propuesto por estos últimos autores (OKSANEN, 1987).

\section{EJEMPLO}

Con el fin de probar este método hemos estudiado las relaciones corológicas y ambientales de ciertos gipsófitos.

La matriz biótica se ha realizado basándose en la cartografía disponible en retículo UTM $10 \times 10 \mathrm{~km}$ de los siguientes táxones gipsícolas, todos ellos elegidos de entre los propuestos por RIVAS-MARTINEZ \& COSTA (1970): Ononis tridentata L., Centaurea hyssopifolia Vahl., Jurinea pinnata DC. y Santolina viscosa Lag. (MOLINA \& al., 1989); Lepidium subulatum L. (RUBIO, 1990); Thymus lacaitae Pau (MORALES, 1986) y Helianthemum squamatum (L.) Pers., Teucrium pumilum L., Teucrium libanitis Schreber, Gypsophila struthium L. subsp struthium y Gypsophila struthium L. subsp. hispanica (Willk.) G. López (RUBIO \& al., 1991). Con estos datos se obtiene un conjunto de 356 cuadrículas de $10 \times 10 \mathrm{~km}$., en cada una de las cuales existe, al menos, una cita cierta de las especies comentadas.

A continuación, y para la elaboración de la matriz abiótica, se procedió a la caracterización de cada una de éstas en función de las variables y con los rangos que se apuntan en la Tabla 1 . Todas las variables se hallan disponibles cartográficamente y se han elegido en relación con su influencia en la fertilidad de los suelos yesíferos (HERNANDO \& al., 1963).

Posteriormente se han sometido los datos a un análisis canónico de correspondencias (-DCCA- TER BRAAK, 1987a). 


\section{VARIABLES ABIÓTICAS}

P: Precipitación media anual

T: Temperatura media anual

M: Temperatura media de las máximas del mes más cálido

m: Temperatura media de las mínimas del mes más frío

ETP: Evapotranspiración media potencial

Y: Tipos de yeso

\section{$\mathbf{P}$}

$1:<300$

2: $300-500$

3: $500-700$

4: $700-1.000$

$5: 1.000-1.500$

$6:<1.500$

m

$1:>6$

2: $6-0$

3: $0--6$

$4:<-6$
T

$1:<6$

2: $6-10$

3: $10-14$

4: $14-18$

5: $>18$

M

$1:<24$

2: $24-30$

3: $30-36$

$4:>36$

\section{ETP}

$1:<600$

2: $600-700$

3: $700-800$

4: $800-900$

$5: 900-1.000$

$6:>1.000$

$\mathbf{Y}$

1: diseminados

2: interestratificados

3: masivos

Tábla 1.- Rangos utilizados con las variables abióticas. Procedencia de la información: Y: MACAU VILAR 1962. P, T, ETP, M y m: FONT TULLOT 1983 P y ETP en mm. T, $\mathbf{M}$ y $\mathbf{m}$ en grados Centígrados.

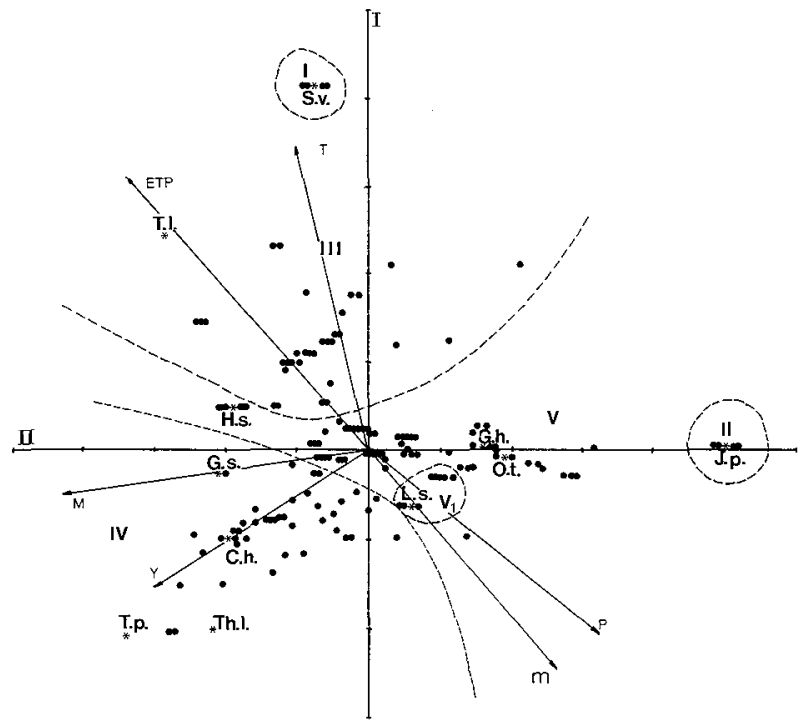

Figura 1.- Plano determinado por los ejes I y II (DCCA) en el que se han individualizado cada uno de los grupos geográficos comentados en el texto (I, II, III, IV, V, V así como representados los vectores ambientales (P,T,M,m,ETP, Y)). (•) cuadrículas UTM de $10 \times 10 \mathrm{~km} .\left(^{*}\right)$ especies utilizadas (O.t. Ononis tridentata; C.h. Centaurea hyssopifolia; J.p. Jurinea pinnata; S.v. Santolina viscosa; L.s. Lepidium subulatum; Th.l. Thymus lacaitae; H.s. Helianthemum squamatum; T.p. Teucrium pumilum; T.I. Teucrium libanitis; G.s. Gypsophila Struthium; G.h. Gypsophila struthium subsp. hispanica). 


\section{RESULTADOS}

Hemos estudiado únicamente el plano I/II (Figura 1) debido a que ambos ejes absorben la mayor parte de la información resultante $(72 \%$ de la varianza). Previamente, y con el fin de comprobar la independencia de las variables ambientales, se calculó la matriz de correlación entre ellas (Tabla 2). Únicamente cabe destacar la elevada correlación positiva de la ETP con la temperatura media y negativa con la temperatura mínima lo cual era de esperar, pero sin que sea lo suficientemente elevada como para aconsejar la eliminación de alguna de las dos.

El primer eje obtenido (Tabla 3) se puede interpretar como un gradiente respecto a los requerimientos hídricos, mientras que el segundo mucho más difícil de interpretar a la luz de las variables consideradas, parece estar influenciado por la participación de las temperaturas extremas. Es interesante señalar la homogeneidad del factor yesos en la extracción de ambos ejes.

Como se puede observar en las Figuras 1 y 2 , únicamente dos grupos se destacan netamente del resto. En el primero (I) se localizan una serie de cuadrículas caracterizadas florísticamente por la presencia exclusiva de Santolina viscosa, en el segundo (II) lo hacen aquellas que sólo presentan

TABLA 2

\begin{tabular}{ccccccc}
\hline $\mathbf{Y}$ & 1.0000 & & & & & \\
ETP & -.0517 & 1.0000 & & & & \\
$\mathbf{T}$ & -.0253 & 0.6692 & 1.0000 & & & \\
$\mathbf{P}$ & -.0088 & -.4663 & -.2957 & 1.0000 & & \\
$\mathbf{M}$ & 0.2088 & 0.3563 & 0.3162 & -.1708 & 1.0000 & \\
$\mathbf{m}$ & -.0816 & -.6177 & -.5472 & 0.3785 & -.2264 & 1.0000 \\
& $\mathbf{Y}$ & $\mathbf{E T P}$ & $\mathbf{T M}$ & $\mathbf{P M}$ & $\mathbf{M}$ & $\mathbf{m}$ \\
\hline
\end{tabular}

Tabla 2.- Matriz de correlación entre las variables ambientales consideradas.

TABLA 3

\begin{tabular}{lcccc}
\hline & $\begin{array}{c}\text { COEFICIENTES CANÓNICOS } \\
\text { eje 1 }\end{array}$ & eje 2 & $\begin{array}{c}\text { COEFICIENTES DE CORRELACIÓN } \\
\text { eje 1 }\end{array}$ & eje 2 \\
\hline YESOS & $-0,12$ & $-0,13$ & $-0,22$ & $-0,22$ \\
ETP & 0,16 & 0,15 & 0,47 & $-0,25$ \\
T & 0,35 & 0,12 & 0,52 & $-0,08$ \\
P & $-0,11$ & 0,09 & $-0,31$ & 0,24 \\
M & $-0,24$ & $-0,19$ & $-0,08$ & $-0,32$ \\
m & $-0,04$ & 0,02 & $-0,37$ & 0,19 \\
\hline
\end{tabular}

Tabla 3.- Coeficientes canónicos y coeficientes de correlación obtenidos en el análisis para las variables respecto a los ejes 1 y 2 . 


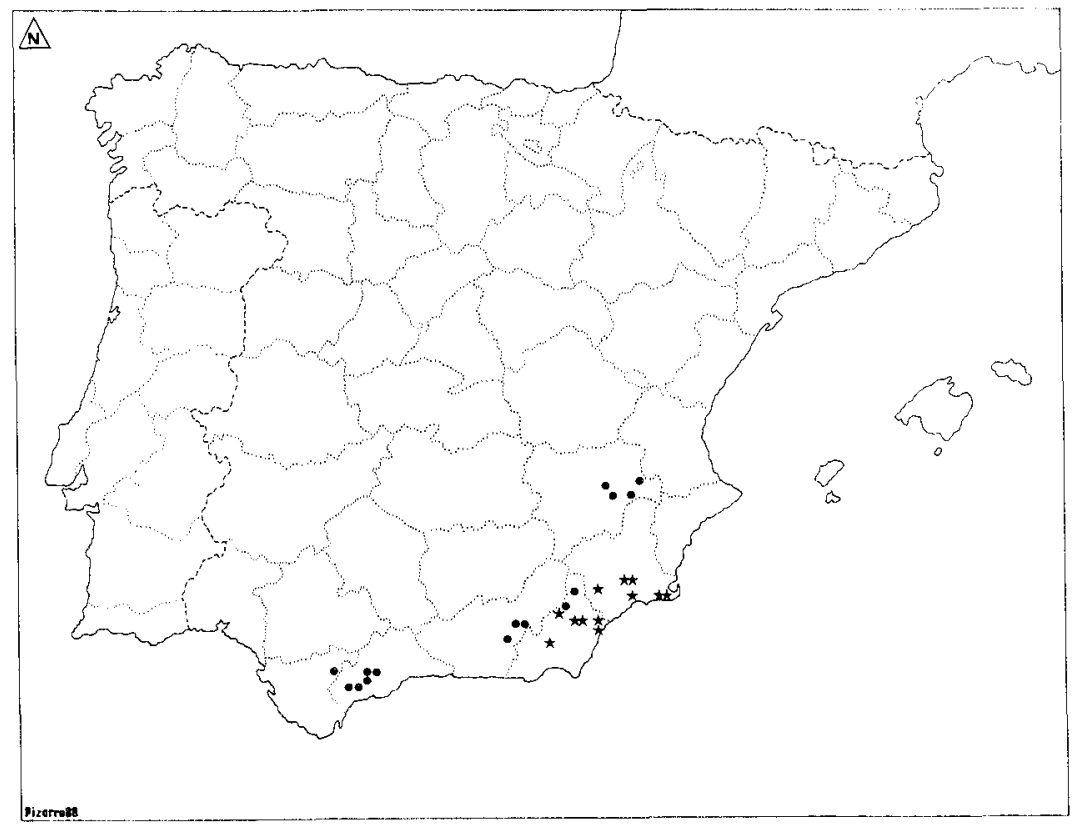

Figura 2.- Representación geográfica de las cuadrículas correspondientes a los grupos I (*) y II $(\bullet)$.

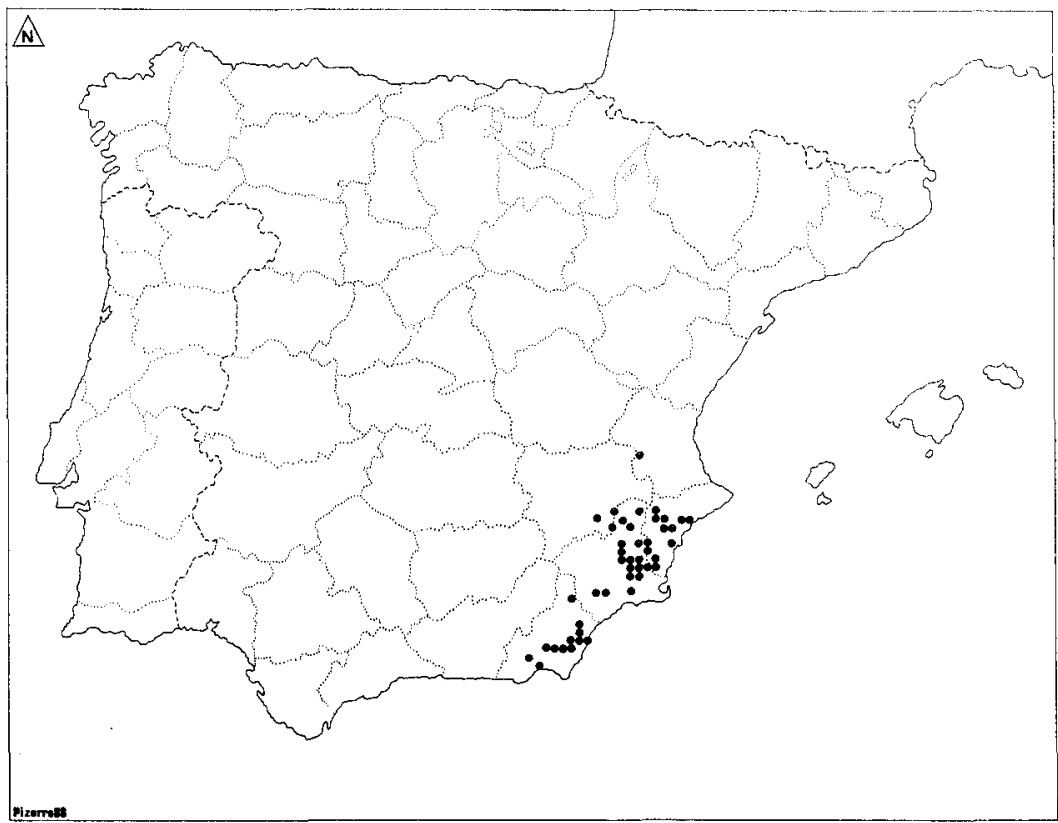

Figura 3.- Representación geográfica de las cuadrículas correspondientes al grupo III. 
Jurinea pinnata. Ambos se sitúan en la parte negativa del gradiente correspondiente a la variable "Tipos de yesos", lo que se corresponde con el carácter subgipsófito de ambos táxones (RIVAS GODAY \& RIVAS-MARTÍNEZ, 1967; RIVAS-MARTÍNEZ \& COSTA, 1970).

Santolina viscosa es un endemismo de los territorios semiáridos de la provincia Murciano-Almeriense (ALCARAZ \& PEINADO, 1987), razón por la que este grupo de cuadrículas se sitúa en el extremo superior de los gradientes de Temperatura media y de Evapotranspiración Potencial. Jurinea pinnata es un taxon endémico de la mitad oriental de la península ibérica al que se le conoce una doble valencia ecológica. Por un lado caracteriza las comunidades yesíferas de la Hoya de Baza, así como a las toledanomanchegas de la cabecera del Algodor, desarrollándose también sobre los yesos de la fosa de Calatayud. Por otro, y siempre a mayor altura, también se desarrolla sobre dolomías cristalinas (Serranía de Ronda) o kakeritizadas (Sierra de Baza) (GÓMEZ MERCADO \& VALLE TENDERO, 1988). Son precisamente las cuadrículas correspondientes a estas últimas sierras las que se reúnen en el grupo II, por lo que al ser la pluviometría de éstas superior a la media del conjunto de los territorios yesíferos ibéricos, hace que este grupo se localice en la zona extrema del eje de precipitación (Figuras 1 y 2 ).

Los tres grupos restantes que pueden diferenciarse con las demás cuadrículas no se separan entre sí de una forma tan clara como los dos primeros, existiendo entre ellos una transición gradual. No obstante los tres tienen un claro significado tanto ecológico, como corológico y fitosociológico.

El grupo III (Figura 3), que se independiza a favor de una elevada temperatura media y altos índices de ETP agrupa a las yeseras termo y mesomediterráneas inferiores del sureste ibérico. Dentro de este grupo se sitúan, por un lado los caracterizados por Teucrium libanitis, incluibles en la alianza Teucrion verticillati, y por otro los correspondientes al Santolino viscosae-Gypsophiletum struthii, del semiárido almeriense, que representa el extremo termomediterráneo, perteneciente a la alianza Lepidion subulati.

El grupo IV es el mejor caracterizado por la presencia de Centaurea hyssopifolia, Thymus lacaitae, Teucrium pumilum. (Figura 4). En él se localizan la práctica totalidad de los yesos manchegos y buena parte de los guadiciano bacenses, lo que se corresponde con el areal mesomediterráneo de la alianza Lepidion subulati. Gypsophila struthium, taxon característico de esta alianza, se localiza en una posición intermedia entre este grupo y el anterior.

El resto de las cuadrículas (grupo V), se localizan en la parte positiva de los gradientes de precipitación y de las temperaturas mínimas. Florísticamente aparece caracterizado por Ononis tridentata, Lepidium subulatum y Gypophila hispanica. Helianthemum squamatum, de apetencias más termófilas que las anteriores ocupa una posición intermedia entre los tres grupos anteriormente definidos. 


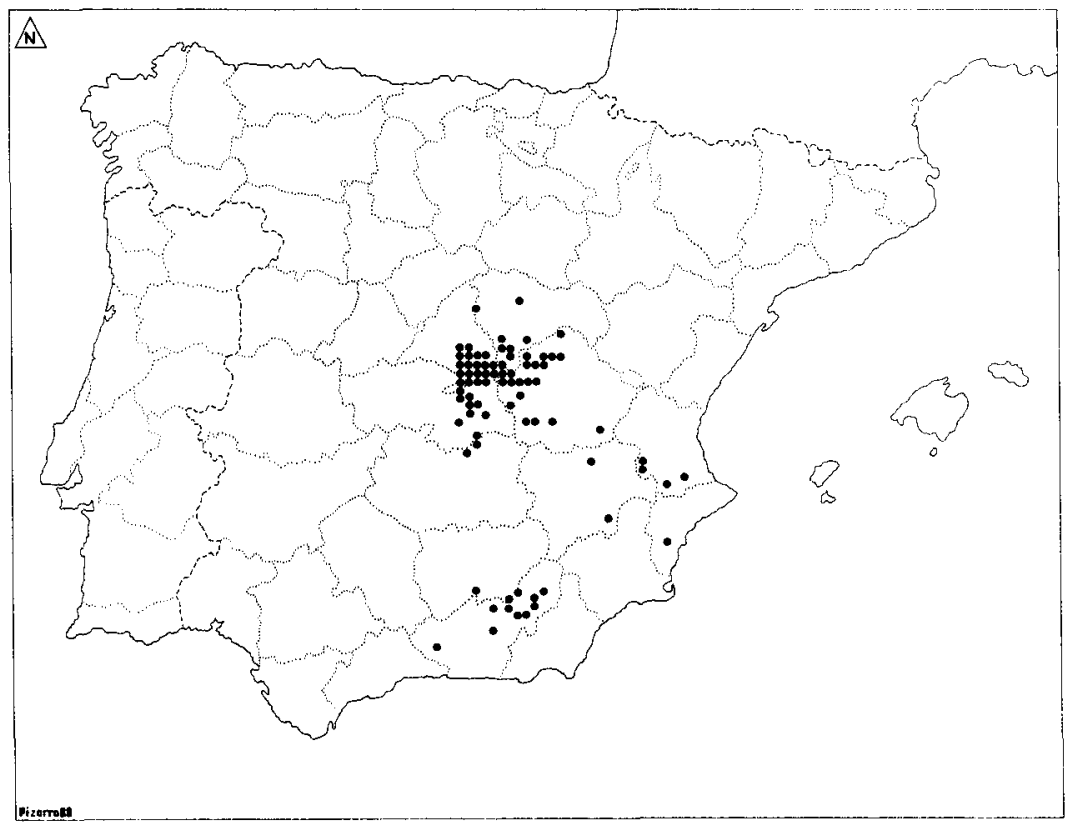

Figura 4.- Representación geográfica de las cuadrículas correspondientes al grupo IV.

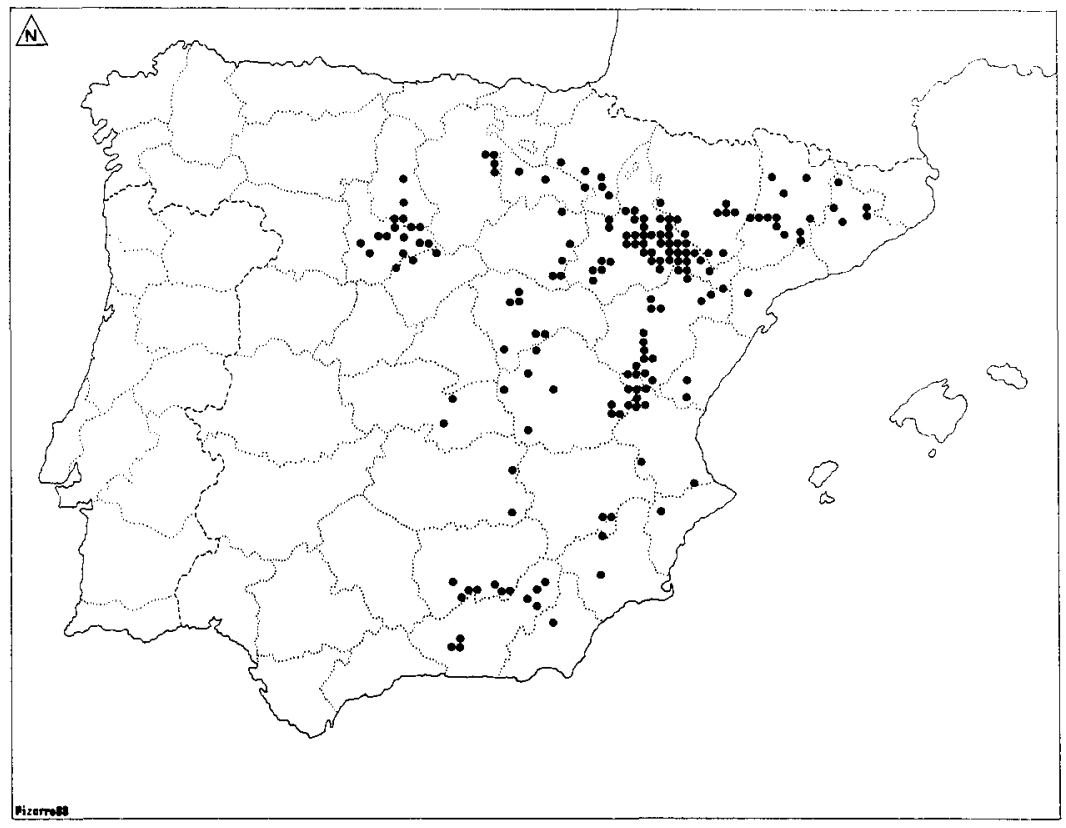

Figura 5.- Representación geográfica de las cuadrículas correspondientes al grupo $\mathrm{V}$ y $\mathrm{V}_{1}$. 


\begin{tabular}{|c|c|c|c|c|}
\hline GRUPOS & $\begin{array}{l}\text { CARACTER } \\
\text { FLORÍSTICOS }\end{array}$ & $\begin{array}{l}\text { CARACTERIZACIÓN } \\
\text { COROLOGÍA }\end{array}$ & $\begin{array}{l}\text { VARACTERIZACIÓN } \\
\text { BIOCLIMÁTICA }\end{array}$ & FITOSOCIOLÓGICA \\
\hline I & Santolina viscosa & Murciano-almeriense & Termo-semiárido & \multirow[b]{2}{*}{$\begin{array}{l}\text { Sintaxones no } \\
\text { incluibles en } \\
\text { Gypsophiletalia }\end{array}$} \\
\hline II & $\begin{array}{l}\text { Malacitano-Almijarense } \\
\text { Jurinea pinnata }\end{array}$ & $\begin{array}{l}\text { Meso-supra } \\
\text { Guadiciano-Bacense } \\
\text { Manchega }\end{array}$ & Seco-Subhúmedo & \\
\hline III & Teucrium libanitis & Murciano-Almeriense & Termo-semiárido & $\begin{array}{l}\text { Teucrion libanitis + } \\
\text { Santolino-Gypsophiletum }\end{array}$ \\
\hline IV & $\begin{array}{l}\text { Gupsophyla struthium } \\
\text { Centaurea hyssopifolia } \\
\text { Teucrium pumilum } \\
\text { Thymus lacaitac }\end{array}$ & $\begin{array}{l}\text { Manchega } \\
\text { Guadiciano-bacense }\end{array}$ & Meso-Seco & Lepidenion subulati \\
\hline$V-V_{1}$ & $\begin{array}{l}\text { Helianthemum squamatum } \\
\text { Ononis tridentata } \\
\text { Gypsophila hispanica } \\
\text { Lepidium subulatum }\end{array}$ & $\begin{array}{l}\text { nAragonés } \\
\text { Castellano-Duriense }\end{array}$ & $\begin{array}{l}\text { Meso-Semiárido }(\mathrm{V}) \\
\text { Meso-Seco }\left(\mathrm{V}_{1}\right)\end{array}$ & $\begin{array}{l}\text { Gypsophilenion } \\
\text { hispanicae }+ \\
\text { Salvion lavandulifoliae, } \\
\text { no incluible en } \\
\text { Gypsophiletalia }\end{array}$ \\
\hline
\end{tabular}

Tabla 4.- Caracterización fiorística, corológica, bioclimática y fitosociológica de los grupos obtenidos en el DCCA. Hemos seguido la clasificación corológica y bioclimática propuesta por RIVAS-MARTÍNEZ (1987).

Dentro de este grupo (Figura 5) podemos distinguir, por un lado, todas las cuadrículas correspondientes a los afloramientos yesíferos castellano-durienses $\left(\mathrm{V}_{1}\right)$. Están caracterizadas por Ononis tridentata y Lepidium subulatum y constituyen unos afloramientos considerablemente más fríos y húmedos que los del resto de los colonizados por el orden Gypsophiletalia (BURGAZ, 1980; MOLINA, 1984), razón por la cual faltan en ellos los elementos más termófilos del orden. De otra parte se sitúan aquí las cuadrículas correspondientes a los territorios aragoneses, que gravitan alrededor de Gypsophila hispanica, único elemento diferencial frente a los yesos manchegos y que, a diferencia de los del grupo $V_{1}$ se sitúan en el centro de la distribución, debido a las condiciones hídricas características de la depresión del Ebro. Otras cuadrículas situadas en este grupo son aquellas pertenecientes a diversas zonas peninsulares, mal caracterizadas florísticamente con la metodología utilizada, y en las que únicamente han sido citados los táxones de más amplia distribución dentro del orden.

En la Tabla 4 se resumen las características florísticas, corológicas y bioclimáticas de los grupos identificados. Asimismo se incluye la caracterización fitosociológica de cada uno de ellos, al menos siempre que ello ha sido posible. Se observa, al comparar con el esquema fitosociológico de los yesos ibéricos, que se adjunta como apéndice, que existe una buena correlación entre los grupos obtenidos y el esquema que actualmente se admite para éstos. 
El esquema fitosociológico reconocido en la actualidad para sistematizar la vegetación gipsícola ibérica, hasta nivel de alianza es el siguiente. Así mismo se incluye también en él las asociaciones citadas en el texto. 1956.

* Gypsophiletalia (Bellot 1952) Bellot \& Rivas Goday in Rivas Goday 1956.

Lepidion subulati (Bellot 1952) Bellot \& Rivs Goday in Rivas Goday

** Lepidenion subulati as. Santolino viscosae-Gypsophiletum struthii Rivas Goday \& Esteve 1965.

** Gypsophilenion hispanicae (Br.-Bl. \& O. Bolòs 1957) Loidi, Fernández-González \& Molina inéd.

* Thymo-Teucrion verticillati Rivas Goday 1956.

En lo referente a la sistematización de los tomillares gipsícolas de la cuenca del Duero y de acuerdo con MOLINA \& IZCO (1988) deben de situarse dentro de la Alianza Sideritido incanae - Salvion lavandulifoliae (Rivas Goday \& Rivas-Martínez 1969) Izco \& Molina 1987, perteneciente al orden Rosmarinetalia Br-Bl. (1931) 1952.

\section{CONCLUSIONES}

En el ejemplo considerado parece evidente que los resultados obtenidos mediante las técnicas consideradas se ajustan de forma satisfactoria, tanto a los conocimientos biogeográficos, como fitosociológicos y ecológicos que sobre las comunidades gipsícolas se tiene en la Península Ibérica.

Por otro lado, los vectores directores son pequeños, lambda $a_{1}=0.23$, lambda $=0.09$, por consiguiente los gradientes extraídos son cortos (GAUCH \& STONE, 1979). Esto quiere decir que los óptimos del algunas especies caen fuera de la región donde se sitúan las cuadrículas. Aunque ello parece implicar una violación de una de las condiciones requeridas por el modelo aplicado, los resultados son satisfactorios en la línea de los obtenidos por TER BRAAK (1986) con idénticas dificultades.

Pese a todo, los coeficientes de correlación especie-variables ambientales (TER BRAAK, 1986) no son tan satisfactorios como desearíamos (Eje 1: 0,65 y Eje 2: 0,45) probablemente se deba a los amplios rangos ambientales considerados (ver Tabla 1) debido a la imposibilidad de disponer de cartografía más detallada.

También respecto a los datos florísticos es conveniente comentar las limitaciones que aparecen. En primer lugar resaltar el hecho de que la cartografía de táxones se realiza de forma positiva. En nuestro caso esto se traduce en la falta de determinados táxones en territorios en donde el cortejo restante de plantas parece indicar la presencia del taxon. Este hecho justifica por sí solo las distorsiones observadas entre las distribuciones de los grupos obtenidos en el análisis frente a la de los sintáxones reconocidos.

En segundo la imposibilidad de cuantificar la presencia de los táxones. En este sentido es interesante resaltar los trabajos de cartografía florística 
de BRISSE \& RUFFRAY (1990) a partir de tablas fitosociológicas lo cual permite valorar la presencia de las plantas en cada lugar.

La profusión de trabajos corológicos y la publicación de mapas de distribución de táxones en series fijas como "Asientos para un atlas corológico de la flora occidental" editada por FERNÁNDEZ CASAS desde 1985 en la revista Fontqueria "Cartografía Corológica Ibérica" editada por MOLINA desde 1989 en Botanica Complutensis, permite plantear trabajos de síntesis geobotánica para los cuales estas técnicas parecen dar buenos resultados.

\section{BIBLIOGRAFÍA}

ALCARAZ, F. \& PEINADO, M., 1987. España semiárida: Murcia y Almería. in PEINADO, M. \& RIVAS-MARTÍNEZ (eds.) "La vegetación de España". Madrid.

BRISSE, H. \& RUFFRAY, P. 1990. Cartografía de la flore et de la vegetation de la France por microordinateur, Proc. X Jornadas de Fitosociología. Granada.

BURGAZ, A. R., 1983. Flora y vegetación gipsófila de la provincia de Valladolid y Sureste de la de Palencia. Valladolid.

ELENA ROSElLÓ, R. \& BUNCE, R. 1984. Aplicación del método ITE a la Península Ibérica: Consideraciones metodológicas y utilidad en la estimación de la productividad forestal. Anales I.N.I.A. ser. Forestal 8: 45-62.

FERNÁNDEZ-CASAS, J. 1985. Asientos para un atlas corológico de la flora occidental. Mapas 1-4, Fontqueria 8: 23-80.

FONT TULLOT, I. 1983. Atlas climático de España. Inst. Nac. Meteorología. Madrid.

GAUCH, H. G. \& STONE, L. 1979. Vegetation and soil pattern in a mesophytic forest at Ithaca, New York. American Midland Naturalest 102: 332-345.

GITTINS, R. 1985. Canonical analysis: a review with applications in ecology. Springer., Heidelberg.

GÓMEZ MERCADO, F. \& VALLE, F., 1988. Mapa de vegetación de la sierra de Baza. Granada.

HERNADO, V., SÁNCHEZ CONDE, M. P. \& CONTRERAS, J., 1963. Influencia de los niveles de yeso y humedad en la fertilidad del suello yesoso. Anales Edf. Fis. Veg. 22: 329.337 . 
HILL, M. O., 1979. DECORANA: a FORTRAN program for detrended correspondence analysis and reciprocal averaging. Section of Ecology and Systematics, Cornell University, Ithaca, N.Y.

HILL, M. O. \& GAUCH, H. G., 1980. Detrended correspondence analysis, an inproved ordination tecnique. Vegetatio 42: 47-58.

HNATIUK, R. J. \& MASTIN, B. R., 1988. Phytogeography of Acacia in Australia in relation climate and species-richness. Aust. J. Bot., 36: 361-383.

IZCO, J. \& MOLINA, A. 1988. Ensayo sintaxonómico de los matorrales calcifilo continentales incluibles en la nueva alianza Sideritido incanae - Salvion lavanduliforliae. Doc. Phytosociologiques 11: 95-109.

JOGMAN, R. H. G., TER BRAAK, C. J. F. \& VAN TOGEREN, O. F. R., 1987. Data analysis in community and landscape ecology. Wageningen.

KENKEL, N. C. \& BOOTH, T. 1987. A comparision of presence-asence resemblance coefficients for use in biogeographical studies, Coenoses 2: 25-30.

MACAU VILAR, F. 1962. Situación de los terrenos yesíferos enEspaña. Servicio geológico de Obras Públicas. Madrid.

MOlinA, A., RUBIO, A. \& ESCUDERO, A., 1989. Aportaciones 1 a 4. in MOLINA, A. (Ed.) Cartografía Corológica Ibérica. Botanica Complutensis 15: 243-274.

MOLINA, A., 1984. Estudio de los matorrales de Xero-Aphylanthion Rivas Goday \& Rivas Martínez (Sideritido-Salvion) Izco \& Molina al. nova). Tesis doctoral inád. U.C.M. Madrid.

MORALES, R., 1986. Taxonomía de los géneros Thymus (excluida la sección serpyllum) y Thymbra en la Península Ibérica. Ruizia 3. Madrid.

MORENO SAIZ. J. C., 1990. Análisis fitogeográfico del endemismo ibérico-balear en monocotiledóneas. Tesis doctoral inéd. U.A.M. Madrid.

OKSANEN, J., 1988. Anote on the occasional inestability off detrending in correspondence analysis. Vegetatio 74: 29-32.

OKSANEN, J. \& HUTTUNEN, P., 1989. Finding a common ordination for several data sets by incividual difference scaling. Vegetatio 83: 137-145.

RAMOS, A. \& AYUSO, E. 1974. El medio ambiente natural: Un esquema metodológico para la planificación de áreas rurales. Bol. Est. Cent. Ecología 3 (6): 50-68.

REY, J. M., 1984. Cartografía automática de especies y el sistema C.U.T.M. Fontqueria 6: $21-32$.

RIVAS MARTÍNEZ, S., 1987. Mapa de las series de vegetación de España. ICONA. Madrid.

RIVAS MARTÍNEZ, S. \& COSTA, M., 1970. Comunidades gipsícolas del centro de España. Anales Inst. Bot. Cavanilles 27: 193-224.

RIVAS GODAY, S. \& RIVAS MARTÍNEZ, S. 1968. Matorrales y tomillares de la Península Ibérica comprendidos en la clase Ononido Rosmarinetea Br.-Bl. 1947. Anales Inst. Bot. Cavanilles 25: 5-201.

RUBIO SÁNCHEZ, A. 1990. Aportación 24. Molina, A. (ed.) Cartografía Corológica Ibérica. Bot. Complutensis 16: 250-256.

SAINZ, H. \& HERNÁNDEZ-BERMEJO, E., 1984. El análisis de semejanza aplicado al estudio de barreras y fronteras fitogeográficas: Su aplicación a la corología y endemoflora ibérica. Anales Jard. Bot. Madrid 40 (2): 421- 432.

TER BRAAK, C. J. F. \& PRENTICE, I. C., 1988. Theory of gradient analysis. Advances in ecological research 18: 271-317.

TER BRAAK, C. J. F., 1986. Canonical correspondence analysis: A new eigenvector technique for multivariate direct gradient analysis. Ecology 67 (5): 1.167-1.179.

TER BRAAK, C. J. F., 1987a. The analysis of vegetation-environment relationships by canonical correspondence analysis. Vegetatio 69: 69-77.

TER BRAAK, C. J. F. 1987b. CANOCO: a FORTRAN program for canonical community ordinations by (partial) (detrending) (canonical) correspondence analysis, principal component analysis and redundancy analysis (versión 2.1). TNO Insst. Appl. Comp. Sci. Wageningen.

WILLIANS, B.K., 1983. Some observations of the use of discriminant analysis in ecology. Ecology 64: 1.283-1.291.

WHITTAKER, R. H., 1967. Gradient analysis in vegetation. Biol. Rev. 42: 207-264.

YOCCOZ, N. \& CHESSEL, D. 1988. Ordination sous contraintes de réleves d'avifaune: éliminations d'effects dans un plan d'observations à deux facteurs (Constrained ordination of bird count data: effects elimination in a two-factor experimental design). C. R. Acad. Sci. Paris (Ser III) 307: 189-194. 